\title{
Systemic sclerosis and the gastrointestinal tract
}

\author{
Irena Walecka \\ Clinical Department of Dermatology, Central Clinical Hospital of the MSWiA, Warsaw, Poland
}

Gastroenterology Rev 2017; 12 (3): 163-168

DOI: https://doi.org/10.5114/pg.2017.70467

Key words: systemic sclerosis, gastrointestinal involvement, autoantibodies.

Address for correspondence: Irena Walecka MD, PhD, Clinical Department of Dermatology, Central Clinical Hospital of the MSWiA, 137 Wołoska St, $02-507$ Warsaw, Poland, phone: +48 50818 13, e-mail: irena.walecka@cskmswia.pl

\begin{abstract}
Systemic sclerosis (SSc) is an autoimmunological disease of unknown origin with complex pathogenesis and multiple organ involvement. It is characterised by vascular and immunological abnormalities leading to fibrosis of the skin and internal organs. It is a rather rare disease with a prevalence of around 20 per 100,000. The disease results in heterogeneous clinical findings and different courses. Systemic sclerosis usually begins with the onset of Raynaud's phenomenon (RP), followed by skin sclerosis and internal organ involvement, although it may appear synchronously with RP. Gastrointestinal involvement is a serious and prevalent complication of SSc, and the oesophagus is the most frequently affected organ. Both limited and diffuse cutaneous SSc involve internal organs, with the involvement of the gastrointestinal tract as a leading cause of morbidity. At present, treatment is mainly symptomatic with no disease-modifying drugs.
\end{abstract}

\section{Introduction}

Systemic sclerosis (SSc) is a rare autoimmunological, multi-organ disease with a prevalence of around 20 per 100,000 . It is characterised by vasculopathy, fibrosis, and inflammation and mostly affects small vessels and connective tissue. This causes loss of vessels and fibrosis of the skin and internal organs (e.g. digestive tract, lungs, and kidneys) to varying extents and with different courses. Usually SSc begins with the onset of Raynaud's phenomenon (RP) (Figure 1), followed by skin sclerosis (Figure 2) and internal organ involvement.

At times, sclerosis manifests synchronously with RP. Risk factors such as sex, age, the presence of specific autoantibodies in the serum, and the extent of skin involvement are associated with the severity of internal organ involvement, which is a significant cause of mortality and morbidity in patients with systemic sclerosis [1].

\section{Pathogenesis of systemic sclerosis}

The pathogenesis of systemic sclerosis is multivariate. The immune system, vasculature, and connective tissue are involved, although the order and influence of these components remain indistinct and are yet to be defined. The disease, results in heterogeneous clinical findings and different courses with various patterns of skin and internal organ involvement [2].
There are numerous publications supporting the hypothesis that unknown environmental factors are essential in the development of scleroderma. Although the presumed factors and mechanisms are yet to be defined, a general idea has been postulated. Unknown factors initiate a sequence of alterations in a susceptible host. They cause endothelial cell injury that selectively affects the microvasculature, followed by fibrosis due to phenotypic conversion of inactive tissue fibroblasts, and epithelial and endothelial cells into activated myofibroblasts.

The role of environmental factors together with genetic factors and epigenetic mechanisms (changes in DNA expression) have been suggested as playing a key role in the pathogenesis of SSc [3].

Anti-centromere autoantibodies (ACA) are the most frequent among patients with systemic sclerosis (15-43\%). These patients more often have pulmonary hypertension whereas digital ulcers (Figure 3), and myocardial and kidney involvement are less frequent. Anti-centromere autoantibodies antibodies are also associated with a limited cutaneous course of disease, a longer time of onset of internal organ involvement, and a lower mortality rate [4]. The presence of ACA antibodies correlates with a better prognosis and longer disease course for SSc patients, although more complications are observed. Other crucial antibodies are anti-topoisomerase I (anti-Scl70) found in 21-34\% of patients with SSc 


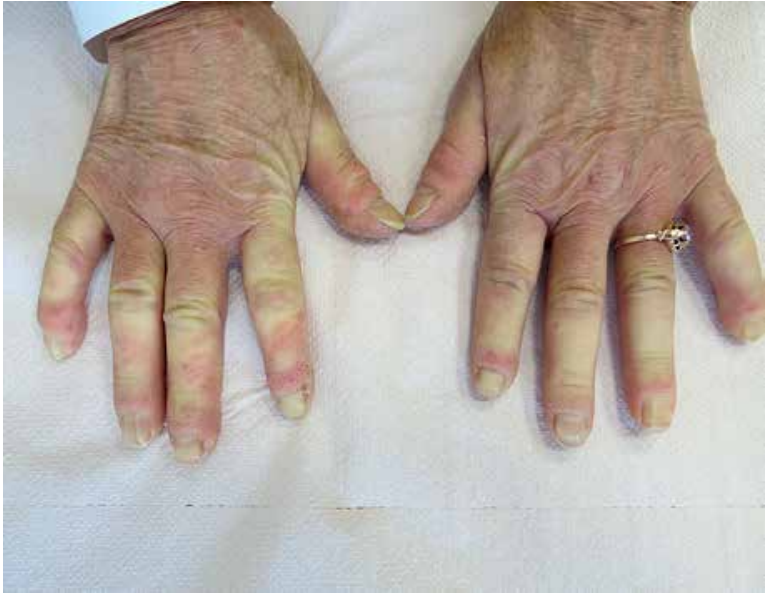

Figure 1. Raynaud's phenomenon in a patient with SSC



Figure 3. Digital ulcers and necrosis in patient with systemic sclerosis

(positive in $47 \%$ of diffuse cutaneous forms vs. $14 \%$ of limited cutaneous forms). Anti-Scl70 are associated with poor prognosis, a higher mortality rate, increased heart and lung involvement, earlier development of RP, and more serious cutaneous forms $[4,5]$.

\section{Gastrointestinal involvement}

As mentioned before, inflammation, vasculopathy, and fibrosis are the main characteristics of SSc and result in a loss of function of the internal organs. Apart from the skin, scleroderma also affects the lungs, heart, kidneys, vascular, and musculoskeletal system, and most commonly involves the gastrointestinal system [6].

Both limited and diffuse cutaneous SSc involve internal organs. Involvement of the gastrointestinal tract (GIT) is the leading cause of morbidity in this disease.

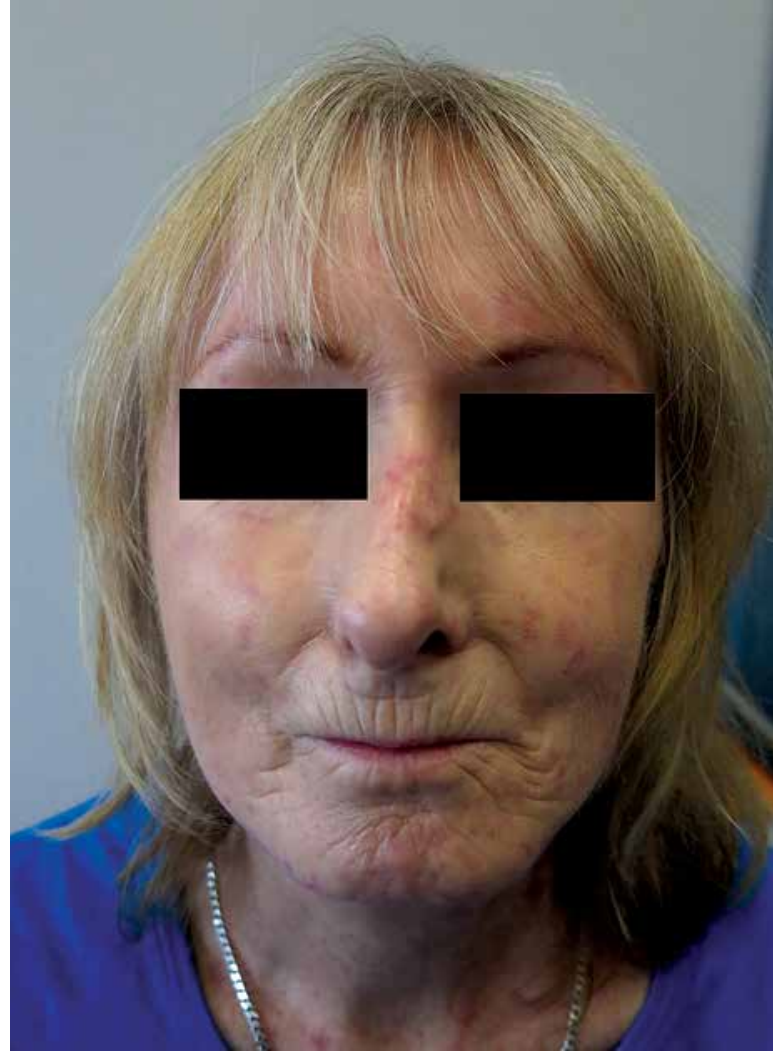

Figure 2. Patient with skin sclerosis and telangiectasias

Oesophageal changes are detected in almost $90 \%$ of patients with SSc. Stomach abnormalities occur in approximately $50 \%$ of subjects and the remaining GI tract disorders affect the small bowel, colon, and anorectal area (in $50-70 \%$ of patients) [7].

Gastrointestinal tract symptoms may occur despite cutaneous changes. They are often non-specific and heterogeneous in clinical manifestations [8]. The pathogenesis of GIT is unclear. Among environmental factors only smoking has been connected to GI flairs [9].

\section{Pathogenesis of GI involvement}

Gastrointestinal tract dysfunctions and structural transformations appear mainly due to fibrosis, and vascular and immune dysfunctions. Diffuse vasculopathy is confirmed by histological findings, e.g. endothelial cell apoptosis and perivascular infiltrations of the stomach and oesophagus [10]. Vascular abnormalities lead to ischaemia followed by neurogenic impairment, which destroys the neurons of the intestinal wall. Next, healthy muscles are replaced with fibrosis causing atrophy [11]. The involvement of the immune system is confirmed by the presence of T-cell infiltrate in the gastric mucosa, causing damage of GIT at an early stage of 
disease [12]. Different fibrogenic cytokines were also detected around the intestinal glands and vessels [13].

Moreover, the role of autoantibodies has also been reported, leading to the conclusion that the presence of Scl-70 and ACA antibodies is less frequent in patients with severe Gl involvement, while the frequency of anti-U3RNP and anti-U1RNP is increased. Despite some speculation, there is no specific antibody specifically related to Gl changes in SSc [14].

\section{Oral cavity}

The main characteristics of oral cavity involvement are microstomia (restriction in mouth opening) and xerostomia (decrease in saliva production). There are few symptoms affecting gingiva and oral mucosa, such as periodontal disease and fragility of the gingiva, taste buds, and oral mucosa, which lead to taste abnormalities [15]. As reported by Pogrel, oral radiological changes show mild to significant osteolysis of the posterior mandibular angle and coronoid process in patients with SSc; however, the exact aetiology is unknown [16].
A therapeutic approach suggests dietary interventions (soft food, eating small pieces together with fluids and oral hygiene) and physical therapy (e.g. mouth stretching).

\section{Oesophagus}

Of the entire GI tract, the oesophagus is the most commonly affected organ. The main symptoms include dysphagia, heartburn and regurgitation, as well as secondary to oesophageal reflux - cough, pharyngitis, or asthma [17]. Oesophageal complications include lower oesophageal sphincter (LOS) abnormalities, gastroesophageal reflux disease (GERD), and Barrett's oesophagus [18].

The reduction in LOS tone (neither opening normally nor closing), together with a decrease or lack of primary peristalsis in the lower oesophagus lead to dilated (Figures 4,5$)$ and patulous oesophagus, resulting in a high probability of GERD (a result of damage to the LOS by allowing gastric acid reflux into the oesophagus). Simple peptic esophagitis initially caused by GERD may


Figure 4. Dilation of oesophagus in a patient with systemic sclerosis 

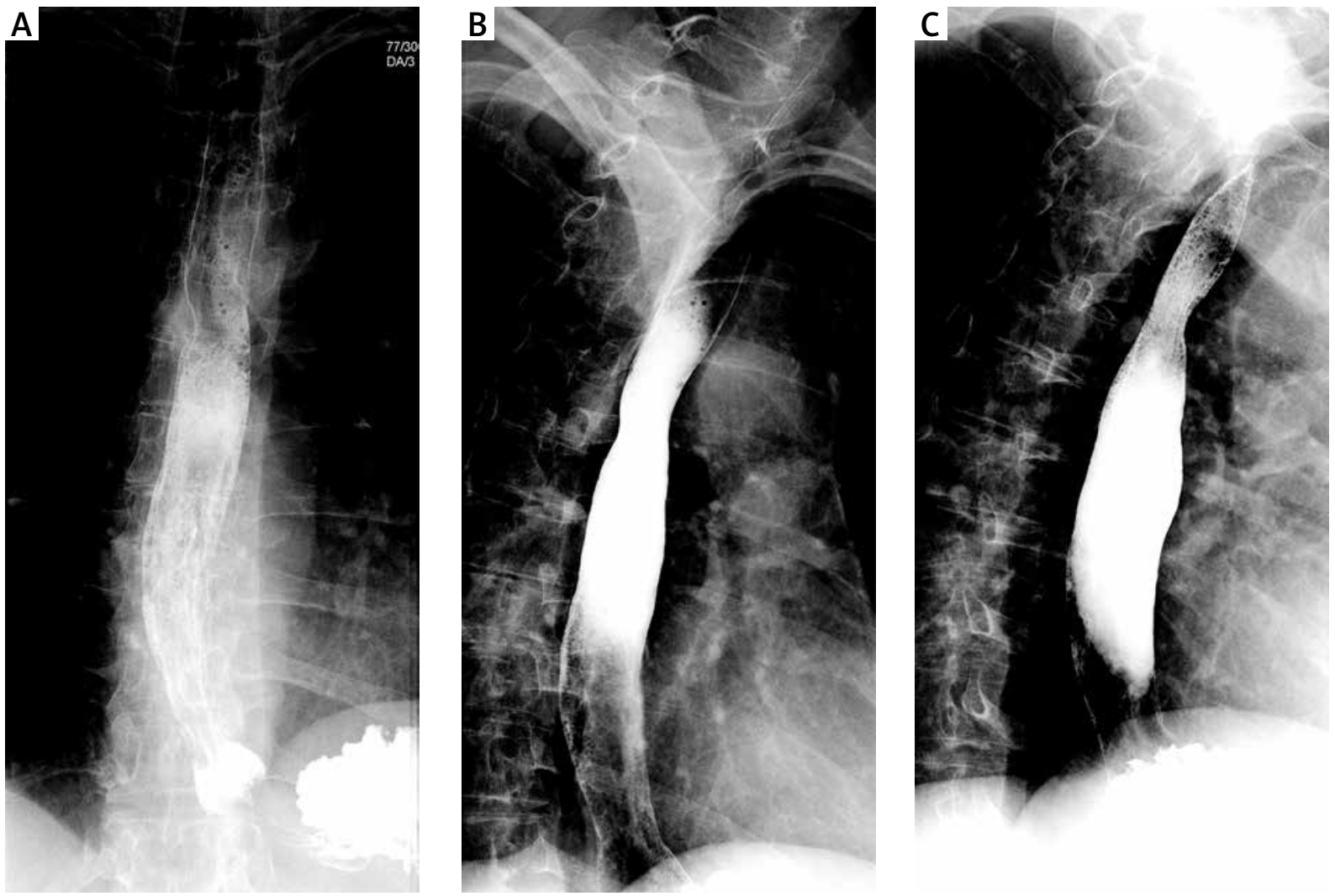

Figure 5. Dilation of oesophagus in a patient with systemic sclerosis

progress to erosive oesophagitis together with bleeding and ulcerations; however, the severity of GERD is not associated with the intensity of symptoms, but rather with pulmonary interstitial fibrosis, probably caused by micro-aspirations of gastric contents. Long-lasting GERD may even lead to Barrett's oesophagus and later on to oesophageal adenocarcinoma [12].

\section{Stomach}

Stomach involvement demonstrates gastric antral vascular ectasia (GAVE; presented in endoscopic features as "watermelon stomach") and gastroparesis. Gastric antral vascular ectasia usually leads to delayed gastric emptying, which manifests with satiety, vomiting, and bloating, followed by eight loss and malnutrition [19]. Under gastroendoscopic observation, GAVE is characterised by parallel longitudinal columns of red vessels among the gastric antrum eradiating to the pylorus, resembling a watermelon [20]. The prevalence of GAVE varies from $5.7 \%$ to $22.3 \%$ of SSc subjects, although in many cases it may be the first manifestation of systemic sclerosis [21]. Gastroparesis, on the other hand, is a condition resulting in chronic motility alternations and delayed emptying of the stomach causing patients to suffer from nausea, vomiting, or dyspepsia.

\section{Small bowel}

The small bowel is involved in around 40\% [22] of SSc patients, with the main clinical features of chronic intestinal pseudo-obstruction (CIPO), pneumatosis cystoides intestinalis, and small intestinal bacterial overgrowth (SIBO) [23].

Systemic sclerosis patients suffer from small bowel motility disturbance leading to obstipation, usually followed by pseudo-obstruction. It is characterized by diversity of symptoms (nausea, vomiting, bloating, diarrhoea) and signs of obstruction with no occluding lesion found. It can be acute or chronic and of various degrees of severity. Therefore, stasis due to dysmotility is a risk factor of developing SIBO [24], which is described as the presence of $1 \times 10^{5} \mathrm{organism} / \mathrm{mm}$ of duodenal aspirate fluid, often leading to malabsorption and malnutrition.

The presence of air cysts in the mucosa and submucosa of the small intestines is called pneumatosis cystoides intestinalis, a rare condition, with a poor prognosis for SSc patients. It is also secondary to dysmotility. Radiography shows multiple air fluid levels and dilation of the bowel. Percutaneous coronary interbvention usually has no serious consequences although, for example, intestinal ischaemia may occur [25]. 


\section{Colon and anus}

Colon and anorectal involvement is present in 10$70 \%$ of SSc patients. Delayed colon transit due to colon hypomotility, telangiectasias causing bleeding, and anaemia are common signs.

Although colonic involvement is often asymptomatic, it may even lead to serious perforation requiring surgical intervention. Due to the co-existence of SIBO causing diarrhoea, constipations in general do not last for long [26].

Patients with anal involvement often suffer from faecal incontinence as a result of constipation with rectal prolapse or internal anal sphincter dysfunction. The development of faecal incontinence has been proven to be secondary to neuropathy and myopathy. Malandrini et al. observed nerve degeneration in the rectal mucosa of SSc patients with systemic sclerosis.

Moreover, deposition of bad collagen breaks neural fibre connections and leads to neuropathy [27].

\section{Diagnosis and management}

The therapy of systemic sclerosis depends on the severity of the disease, organ involvement, and its progression. It is determined on an individual basis, depending on the clinical symptoms and targeted vasculature, connective tissue involvement, and the immune system [28].

Every SSc patient, despite the presence or absence of gastric symptoms, should be referred for gastroenterological care. Patients are required to undergo various tests, radiological and endoscopic imaging, together with gastroenterological consultations. The treatment of gastroenterological complications is mainly symptomatic and supportive, with no disease-modifying drugs at present.

Patients are given acid-reducing therapy, cyclic antibiotics, and pro-kinetic agents. Immunosuppressive therapy targeting fibrosis gives hope for future success [29].

Symptoms of dysmotility have a great impact on the quality of life of patients with SSc. Diarrhoea, nausea, or weight loss may even result in severe malnutrition. Therefore, the ultimate goal is to improve the nutritional status of SSc patients.

Acid suppression treatment - recommended twice daily; proton pump inhibitors as basic therapy for GERD. In case of more severe symptoms (such as night-time breakthrough acid reflux) H2-blockers may be needed.

Nutritional intervention - commence therapy for gastroparesis and introduce multiple small, low-fibre meals. In case of a delay in gastric emptying, pro-kinetic medications may be required.
Antibiotics - in case of the occurrence of small intestinal bacterial overgrowth. Rifaximin is recommended and is fairly new and safe with few systemic side effects [30].

Endotherapy (argon plasma coagulation) - for treatment of gastric antral vascular ectasia [31].

Immune suppression (cyclophosphamide or mycophenolate mofetil) - may cause considerable improvement in patients with early diffuse progressive disease [32].

Immunomodulatory treatment (intravenous immunoglobulins) - relatively safe and could be potentially beneficial by neutralising muscarinic autoantibodies and causing a reduction in pro-fibrotic cytokines [33].

Complex approach to faecal incontinence - focuses on behavioural therapies and management of diarrhoea. Combined medical, physical, and nutritional therapies or new sacral nerve stimulation can contribute to improved quality of life [34].

\section{Discussion}

Due to the involvement of the skin and internal organs (especially the gastrointestinal tract), systemic sclerosis appears to be an interdisciplinary disease. Therefore, cooperation between different specialists is crucial. Due to highly frequent Gl involvement, SSc patients require regular and long-term gastroenterological care. In view of the slow progression of the disease in general, closer cooperation between specialists seems to be essential in introducing early and effective therapy and preventing short- and long-term complications.

\section{Summary}

Gastrointestinal involvement is common in patients with systemic sclerosis. Symptoms may occur at any stage of the disease, sometimes appearing long before skin involvement. The most commonly affected organ is the oesophagus, usually manifesting as dysphagia, heartburn, and regurgitation. Early diagnosis is crucial for improving prognosis and slowing down the course of the disease through proper treatment.

\section{Conflict of interest}

The author declares no conflict of interest.

\section{References}

1. Szymanska E, Wieczorek M, Lagun Z. Vascular changes in autoimmunological connective tissue diseases. Acta Angiol 2016; 22: 172-6.

2. Sticherling M. Systemic sclerosis - dermatological aspects. Part 1: Pathogenesis, epidemiology, clinical findings. Prof. Dr. Jan C. Simon, Leipzig (eds). JDDG 2012; 10: 705-16. 
3. Manetti $M$, Matucii-Cerinic $M$. The new frontier in systemic sclerosis: from epigenetics to new treatments. Rheumatology 2015; 54: 1757-8.

4. Mehra S, Walker J, Patterson K, Fritzler MJ. Autoantibodies in systemic sclerosis. Autoimmun Rev 2013; 12: 340-54.

5. Sobanski V, Dauchet L, Lefèvre G, et al. Prevalence of anti-RNA polymerase III antibodies in systemic sclerosis: new data from a French cohort and a systematic review and meta-analysis: prevalence of anti-RNAP III in SSc. Arthritis Rheumatol 2014; 66: 407-17.

6. Tay T, Ferdowsi N, Baron M, et al. Measures of disease status in systemic sclerosis: a systematic review. Semin Arthr Rheum 2017; 46: 473-87.

7. Braun-Moscovici Y, Brun R, Braun M. Systemic sclerosis and the gastrointestinal tract - clinical approach. Rambam Maimonides Med J 2016; 7: e0031.

8. Steen VD, Medsger TA Jr. Severe organ involvement in systemic sclerosis with diffuse scleroderma. Arthritis Rheum 2000; 43 2437-44.

9. Hudson M, Lo E, Lu Y, et al. Cigarette smoking in patients with systemic sclerosis. Arthritis Rheum 2011; 63: 230-8.

10. Cohen S, Fisher R, Lipshutz W, et al. The pathogenesis of esophageal dysfunction in scleroderma and Raynaud's disease. J Clin Investig 1972; 51: 2663-8.

11. Schmeiser T, Saar P, Jin D, et al. Profile of gastrointestinal involvement in patients with systemic sclerosis. Rheumatol Int 2012; 32: 2471-8

12. Tiev KP, Cabane J. Digestive tract involvement in systemic sclerosis. Autoimmun Rev 2011; 11: 68-73.

13. Zuber-Jerger I, Müller A, Kullmann F, et al. Gastrointestinal manifestation of systemic sclerosis: thickening of the upper gastrointestinal wall detected by endoscopic ultrasound is a valid sign. Rheumatology (Oxford) 2010; 49: 368-72.

14. Nishimagi E, Tochimoto A, Kawaguchi Y, et al. Characteristics of patients with early systemic sclerosis and severe gastrointestinal tract involvement. J Rheumatol 2007; 34: 2050-5.

15. Attar A. Digestive manifestations in systemic sclerosis. Ann Med Intern 2002; 153: 260-4

16. Pogrel MA. Unilateral osteolysis of the mandibular angle and coronoid process in scleroderma. Int J Oral Maxillofac Surg 1988; 17: 155-6.

17. Raja J, Ng CT, Sujau I, et al. High-resolution oesophageal manometry and 24-hour impedance-pH study in systemic sclerosis patients: association with clinical features, symptoms and severity. Clin Exp Rheumatol 2016; 34 (5 Suppl. 100): 115-21.

18. Ntoumazios SK, Voulgari PV, Potsis K, et al. Esophageal involvement in scleroderma: gastroesophageal reflux, the common problem. Semin Arthritis Rheum 2006; 36: 173-81.

19. Marie I, Gourcerol G, Leroi AM, et al. Delayed gastric emptying determined using the 13C-octanoic acid breath test in patients with systemic sclerosis. Arthritis Rheum 2012; 64: 2346-55.

20. Elkayam O, Oumanski M, Yaron M, Caspi D. Watermelon stomach following and preceding systemic sclerosis. Semin Arthritis Rheum 2000; 30: 127-31.

21. Hung EW, Mayes MD, Sharif R, et al. Gastric antral vascular ectasia and its clinical correlates in patients with early diffuse systemic sclerosis in the SCOT trial. J Rheumatol 2013; 40: 455-60.
22. Fynne L, Worsøe J, Gregersen T, et al. Gastrointestinal transit in patients with systemic sclerosis. Scand J Gastroenterol 2011; 46: 1187-93.

23. Gregersen H, Liao D, Pedersen J, Drewes AM. A new method for evaluation of intestinal muscle contraction properties: studies in normal subjects and in patients with systemic sclerosis. Neurogastroenterol Motil 2007; 19: 11-9.

24. Valenzuela A, Li S, Becker L, et al. Intestinal pseudo-obstruction in patients with systemic sclerosis: an analysis of the nationwide inpatient sample. Rheumatology (Oxford) 2016; 55 : 654-8.

25. Kaneko M, Sasaki S, Teruya S, et al. Pneumatosis cystoides intestinalis in patients with systemic sclerosis: a case report and review of 39 Japanese cases. Case Rep Gastrointest Med 2016; 2016: 2474515.

26. Richard N, Hudson M, Gyger G, et al. Clinical correlates of faecal incontinence in systemic sclerosis: identifying therapeutic avenues. Rheumatology 2017; 56: 581-8.

27. Malandrini A, Selvi E, Villanova $M$, et al. Autonomic nervous system and smooth muscle cell involvement in systemic sclerosis: ultrastructural study of 3 cases. J Rheumatol 2000; 27: 1203-6.

28. Steen VD. Targeted therapy for systemic sclerosis. Autoimmun Rev 2006; 5: 122-4.

29. Frech TM, Khanna D, Maranian P, et al. Probiotics for the treatment of systemic sclerosis-associated gastrointestinal bloating/distention. Clin Exp Rheumatol 2011; 29 (2 Suppl 65): S22-25.

30. Nikou GC, Toumpanakis C, Katsiari C, et al. Treatment of small intestinal disease in systemic sclerosis with octreotide: a prospective study in seven patients. J Clin Rheumatol 2007; 13 : 119-23.

31. Marie I, Ducrotte P, Antonietti M, et al. Watermelon stomach in systemic sclerosis: its incidence and management. Aliment Pharmacol Ther 2008; 28: 412-21.

32. Papachristos DA, Nikpour M, Hair C, Stevens WM. Intravenous cyclophosphamide as a therapeutic option for severe refractory gastric antral vascular ectasia in systemic sclerosis. Intern Med J 2015; 45: 1077-81.

33. Raja J, Nihtyanova SI, Murray CD, et al. Sustained benefit from intravenous immunoglobulin therapy for gastrointestinal involvement in systemic sclerosis. Rheumatology (Oxford) 2016; 55: 115-9.

34. Burr SK, Alam A, Cohen R, et al. Lack of effect of sacral nerve stimulation for incontinence in patients with systemic sclerosis. Colorectal Dis 2015; 17: 903-7.

Received: 25.04.2017

Accepted: 1.06 .2017 